\title{
Association between dysglycemia and mortality by diabetes status and risk factors of dysglycemia in critically ill patients: a retrospective study
}

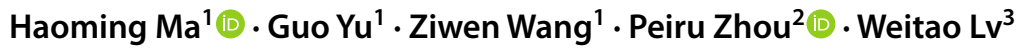

Received: 30 September 2021 / Accepted: 18 October 2021 / Published online: 11 November 2021

(c) The Author(s) 2021, corrected publication 2022

\begin{abstract}
Aims Dysglycemia, including the three domains hyperglycemia, hypoglycemia, and increased glycemic variability (GV), is associated with high mortality among critically ill patients. However, this association differs by diabetes status, and reports in this regard are limited. This study aimed to evaluate the associations between the three dysglycemia domains and mortality in critically ill patients by diabetes status and determined the contributing factors for dysglycemia.

Methods This retrospective study included 958 critically ill patients (admitted to the ICU) with or without DM. Dysglycemia was defined as abnormality of any of the three dimensions. We evaluated the effects of the three domains of glucose control on mortality using binary logistic regression and then adjusted for confounders. The associations between dysglycemia and other variables were investigated using cumulative logistic regression analysis.

Result GV independently and similarly affected mortality in both groups after adjustment for confounders (DM: odds ratio [OR], 1.05; 95\% confidence interval [CI]: 1.03-1.08; $p<0.001$; non-DM: OR, 1.07; 95\% CI, 1.03-1.11; $p=0.002$ ). Hypoglycemia was strongly associated with ICU mortality among patients without DM $(3.12 ; 1.76-5.53 ; p<0.001)$ and less so among those with DM $(1.18 ; 0.49-2.83 ; p=0.72)$. Hyperglycemia was non-significantly associated with mortality in both groups. However, the effects of dysglycemia seemed cumulative. The factors contributing to dysglycemia included disease severity, insulin treatment, glucocorticoid use, serum albumin level, total parenteral nutrition, duration of diabetes, elevated procalcitonin level, and need for mechanical ventilation and renal replacement therapy.

Conclusion The association between the three dimensions of dysglycemia and mortality varied by diabetes status. Dysglycemia in critical patients is associated with excess mortality; however, glucose management in patients should be specific to the patient's need considering the diabetes status and broader dimensions. The identified factors for dysglycemia could be used for risk assessment in glucose management requirement in critically ill patients, which may improve clinical outcomes.
\end{abstract}

Keywords Dysglycemia $\cdot$ Diabetes mellitus $\cdot$ Mortality $\cdot$ ICU $\cdot$ Critically ill $\cdot$ Risk factors

\section{Background}

Managed by Massimo Porta.

Haoming Ma and Guo Yu contributed equally to this manuscript.

Peiru Zhou

peiruzhou@jnu.edu.cn

Weitao Lv

13570035005@139.com

1 School of Nursing, Jinan University, No. 601, West Huangpu Avenue, Tianhe District, Guangzhou City, Guangdong Province, China
Most studies have reported on the high incidence of dysglycemia (including the 3 dimensions hyperglycemia, hypoglycemia, and increased glycemic variability $[\mathrm{GV}]$ ) and its

2 Health Management Centre, The First Affiliated Hospital of Jinan University, No. 613, West Huangpu Avenue, Tianhe District, Guangzhou City, Guangdong Province, China

3 Division of Critical Care, The First Affiliated Hospital of Jinan University, No. 613, West Huangpu Avenue, Tianhe District, Guangzhou City, Guangdong Province, China 
independent association with mortality among critically ill patients [1-6]. Recent studies have shown that diabetes status modulates the association between these glycemia domains and mortality or other important clinical outcomes during intensive care unit (ICU) hospitalization [7-12].

The associations between mortality and the 3 domains of dysglycemia have been reported to vary significantly between patients with or without diabetes mellitus (DM) $[7,13]$. Recent observational studies suggest that those with pre-existing diabetes present a "blunted effect" to increased GV and hyperglycemia. This has been attributed to the likely higher tolerance to acute glucose fluctuation in patients with DM [13-15]. However, some recent studies have reported that the association between hypoglycemia and mortality is stronger in patients with DM [7], which is inconsistent with the findings of other studies. Moreover, most of these studies did not consider comorbidities, disease severity, inflammation level, insulin therapy and other in ICU treatments (including glucocorticoid use and nutrition therapy), which known as confounding factors when estimating the effects of glucose metrics [16].

Therefore, the associations between different glycemic metrics and mortality in ICU remain unclear, and studies directly and quantitatively comparing the effects of these glucose control domains in critically ill patients with comprehensive adjustments for confounders are scarce [17]. Because the complex association between ICU patients' outcomes and GV depends on DM, determining the effects of different glycemic metrics in DM and non-DM patients is important.

In this context, we hypothesized that the effects of the 3 domains of dysglycemia on mortality would differ between patients with or without DM. Therefore, we conducted a retrospective study using data of ICU patients to, primarily, explore the effect of different glycemic metrics on mortality and further identify risk factors for dysglycemia in our patient sample.

\section{Methods}

\section{Description of study design and patient enrollment criteria}

This retrospective observational study used electronic clinical data from patients admitted to the ICU of the First Affiliated Hospital, Jinan University between January 1, 2019, and December 31,2020. The institute's ICU is a mixed medical and surgical ICU. Blood glucose level was measured using a glucometer, and measurements were fed into an interactive database.

Patients admitted during the study period who were aged $\geq 18$ years and were treated in the ICU for $\geq 24 \mathrm{~h}$ were eligible. Exclusion criteria included (1) incomplete mortality data; (2) ICU hospitalization $<24 \mathrm{~h}$; (3) $<8$ blood glucose measurements on the first day after admission; (4) treatment discontinuation by family; (5) hospital stay $>120$ days; (6) hospitalization for treatment of diabetic ketoacidosis or diabetic hyperglycemic hyperosmolar state.

\section{Clinical data gathered}

We obtained clinically relevant information from the ICU database and the hospital electronic medical record system. Measures extracted for the analyses included baseline demographics (age, sex, patient diagnosis, and comorbidities, past medical history, severity of illness score [APACHE II score]); ICU treatments (exogenous insulin, nutrition type and steroid use); clinical information (length of ICU stay [ICU-LOS], total length of hospital stay [LOS], blood glucose level, hemodialysis duration in hours, duration of mechanical ventilation, other biochemical indicators, and patient outcomes).

\section{Blood glucose and nutrition management protocol}

According to the guidelines of the American Diabetes Association [18], the target blood glucose range for critically ill patients was $7.8-10.0 \mathrm{mmol} / \mathrm{L}(140-180 \mathrm{mg} / \mathrm{dL})$ in our study. A standard blood glucose control goal and a uniform insulin infusion standard to maintain the blood glucose level within the target range were adopted. The nutrition therapy emphasized on early enteral nutrition, starting feeding within $48 \mathrm{~h}$, and attempting to achieve sufficient nutritional support, in accordance with the recommendations of the ICU nutritional therapist, within 48-72 h [19].

Capillary blood glucose levels were used in this study. The glucose level was measured uniformly by nurses using a glucose meter (FreeStyle Optium Blood Glucose and Ketone Monitoring System, Abbott Diabetes Care, Oxon, UK). Glucose level measurements were made at least every $4 \mathrm{~h}$ after admission to the ICU and for no less than 6 times per day.

\section{Variable definitions}

Patients were assigned to the DM or non-DM group according to the relevant clinical data obtained from patients, family members, and the documentation in their electronic medical records. We also collected the duration of DM (years) for the DM group, which was defined as the time (years) since the first diagnosis of diabetes. Disease severity was assessed using the APACHE II scores [20]. Blood glucose levels during ICU hospitalization were retrieved electronically from the relevant data storage unit, and we used Microsoft Excel software (Microsoft ${ }^{\circledR}$ Excel ${ }^{\circledR} 2016$ MSO 16.0.14131.20278) to calculate the coefficient of variation 
(CV), mean blood glucose (MBG), and standard deviation (SD) for each patient.

We defined the glycemic metrics basis prior studies [21-23]. GV was expressed as the CV of blood glucose in this study [24]. CV is defined as the SD of blood glucose divided by the corresponding MBG (SDIMBG $\times 100)$. We defined increased $\mathrm{GV}$ as $\mathrm{CV} \geq 20 \%$, hypoglycemia as at least $1 \mathrm{BG}$ concentration $<3.9 \mathrm{mmol} / \mathrm{L}(70 \mathrm{mg} / \mathrm{dL})$, and hyperglycemia as mean glucose concentration $>7.8 \mathrm{mmol} / \mathrm{L}$ for non-DM patients and $>10 \mathrm{mmol} / \mathrm{L}$ for $\mathrm{DM}$ patients. Furthermore, dysglycemia was defined as abnormality of any of the 3 dimensions of glucose management (high BG, low BG, and BG excursion). The factors associated with dysglycemia in each patient were defined as ordinal categorical variables, wherein " 0 " referred to no dysglycemia and " 3 " to the presence of all 3 domains of dysglycemia.

\section{Outcome measures and adjustment for confounders}

Hospital mortality, defined as death before hospital discharge, was the main outcome measure in this study. The secondary outcome measure was dysglycemia during ICU stay, including the 3 dimensions of poor glucose management (hyperglycemia, hypoglycemia, increased GV).

Variables considered for adjustment when determining the effect of dysglycemia on adverse outcomes in ICU patients included basic patient information (sex, age, disease severity [basis APACHE II score], MBG, GV [basis $\mathrm{CV}]$, hyperglycemia, hypoglycemia); laboratory data on admission to ICU (white blood cell count, hemoglobin, serum albumin, serum creatinine, and procalcitonin levels); comorbidities (hypertension, coronary heart disease, cerebral infarction, and chronic kidney disease); in ICU treatment (duration of ventilation in hours, duration of hemodialysis in hours, insulin treatment, corticoid use, and nutrition therapy).

\section{Data analysis strategy}

First, we assessed the data distribution. For continuous variables, we used means \pm standard deviations for normally distributed ones and medians and interquartile ranges (25\% quartile to $75 \%$ quartile) for non-normally distributed ones. Categorical variables were presented as percentages.

For between-group comparisons of continuous variables, the t-test was applied to compare the normally distributed variables, whereas the Mann-Whitney rank-sum test was used for non-normally distributed ones. The chi-square test was used to compare categorical variables between groups, and the Bonferroni test was used for pairwise comparisons between groups of 3 or more.

Our patient samples were stratified into 2 groups: critically ill patients with diabetes (DM group) and without diabetes (non-DM group). We performed logistic regression analysis in 2 groups to adjust for age, sex, APACHE II score, comorbidities and laboratory data when estimating the different effects of 3 glycemic metrics (hyperglycemia, hypoglycemia, and glycemic variability) on mortality. For further control of confounders, we adjusted ICU treatment (insulin infusion therapy, glucocorticoid use, duration of ventilation and duration of hemodialysis) and the other two domains of glucose metrics.

The associations between dysglycemia and other variables were investigated using a cumulative logits model, which is suitable for analysis when the dependent variable was an ordinal categorical variable. All significant risk factors $(p<0.2)$ identified in the univariate analysis were considered as potential predictors and then entered into an initial multivariate model. We use forward stepwise selection to enter the independent variables into the model. A 2-sided $p<0.05$ was considered statistically significant. Estimation of effects from the models devised are presented as odds ratios (ORs) and the corresponding 95\% confidence intervals (CIs). We used SPSS version 17.0 software (SPSS Inc., Chicago, IL) for statical analysis.

\section{Results}

\section{Clinical characteristics}

This study included 958 critically ill patients. Figure 1 presents a flowchart for patient screening. Table 1 summarizes the key clinical characteristics of the patients, categorized by ICU mortality. Briefly, the mean patient age was $62.48 \pm 17.86$ years, $613(64.0 \%)$ patients were male, and 238 (24.8\%) patients had DM. The diagnosis in ICU admissions were 245 (25.6\%) for surgical reasons and $713(74,4 \%)$ for medical reasons, with mean APACHE II score was $25.61 \pm 9.15$ at admission. At the end of the study period,

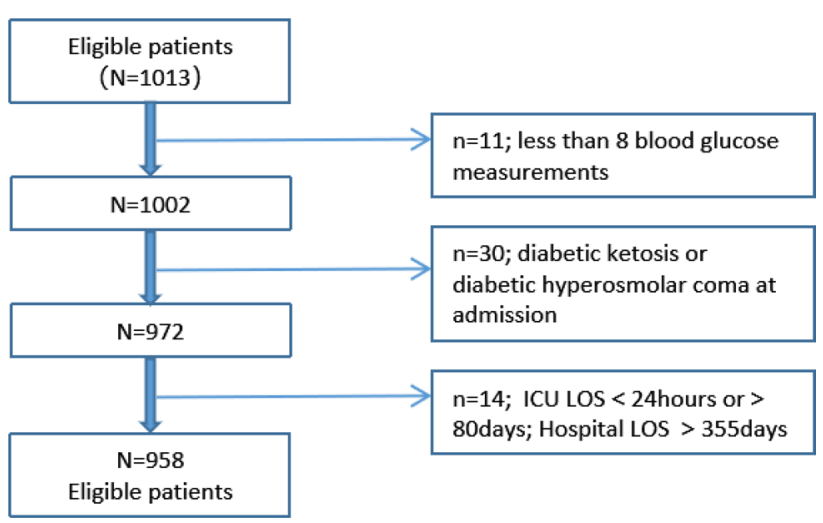

Fig. 1 Patient flow diagram 
Table 1 Patient characteristics

All patients $n=958$

Survivors $n=630$

Dead $n=328$

$P$ value for survivors vs dead in ICU

\begin{tabular}{|c|c|c|c|c|}
\hline \multicolumn{5}{|l|}{ Basic information and glycemia data } \\
\hline Age (years) & $62.48 \pm 17.86$ & $63.07 \pm 17.92$ & $61.34 \pm 17.73$ & 0.154 \\
\hline Male n/N (\%) & $613 / 958(64.0)$ & $407 / 630(64.6)$ & $206 / 328(62.8)$ & 0.79 \\
\hline APACHE II score & $25.61 \pm 9.15$ & $22.85 \pm 8.10$ & $30.93 \pm 8.69$ & $<0.001$ \\
\hline $\mathrm{MBG}(\mathrm{mmol} / \mathrm{L})$ & $10.26 \pm 2.71$ & $9.99 \pm 2.56$ & $10.78 \pm 2.92$ & $<0.001$ \\
\hline SD & $2.61 \pm 1.43$ & $2.28 \pm 1.15$ & $3.25 \pm 1.69$ & $<0.001$ \\
\hline $\mathrm{CV}$ & $24.58 \pm 9.15$ & $22.14 \pm 7.58$ & $29.36 \pm 10.07$ & $<0.001$ \\
\hline Hyperglycemia & $747 / 958(78.0)$ & $479 / 630(76.0)$ & $268 / 328(81.7)$ & 0.044 \\
\hline Hypoglycemia & $178 / 958(18.6)$ & $61 / 630(9.7)$ & $117 / 328(35.7)$ & $<0.001$ \\
\hline \multicolumn{5}{|l|}{ Comorbidities } \\
\hline $\mathrm{DM}(\%)$ & $238 / 958(24.8)$ & $145 / 630(23.0)$ & $93 / 328(28.4)$ & 0.07 \\
\hline Hypertension (\%) & $428 / 958(44.7)$ & $263 / 630(41.7)$ & $165 / 328(50.3)$ & 0.011 \\
\hline $\mathrm{CI}(\%)$ & $155 / 958(16.2)$ & $92 / 630(14.6)$ & $63 / 328(19.2)$ & 0.066 \\
\hline CKD $(\%)$ & $134 / 958(14.0)$ & $74 / 630(11.7)$ & $60 / 328(18.3)$ & 0.006 \\
\hline $\mathrm{CHD}(\%)$ & $146 / 958(15.2)$ & $80 / 630(12.7)$ & $66 / 328(20.1)$ & 0.002 \\
\hline \multicolumn{5}{|l|}{ Clinical data } \\
\hline HOSP-LOS (days) & $16(9-28)$ & $18(11-30)$ & $11(5-23)$ & $<0.001$ \\
\hline ICU-LOS (days) & $5(2-11)$ & $4(2-11)$ & $6(3-12)$ & 0.103 \\
\hline Duration of ventilation (hours) & $24(0-105)$ & $8(0-63)$ & $67(20-171)$ & $<0.001$ \\
\hline Duration of hemodialysis (hours) & $0(0-8)$ & $0(0-4)$ & $0(0-16)$ & $<0.001$ \\
\hline \multicolumn{5}{|l|}{ In ICU treatment } \\
\hline Insulin infusion therapy (\%) & $372 / 958(38.8)$ & $210 / 630(33.3)$ & $162 / 328(49.4)$ & $<0.001$ \\
\hline Use of corticoids (\%) & $381 / 958(39.8)$ & $236 / 630(37.5)$ & $145 / 328(44.2)$ & 0.043 \\
\hline TEN $(\%)$ & $68 / 958(7.1)$ & $52 / 630(8.3)$ & $16 / 328(4.9)$ & 0.054 \\
\hline TPN $(\%)$ & $391 / 958(40.8)$ & $255 / 630(40.5)$ & $136 / 328(41.5)$ & 0.768 \\
\hline $\mathrm{EN}+\mathrm{PN}(\%)$ & $499 / 958(52.1)$ & $323 / 630(51.3)$ & $176 / 328(53.7)$ & 0.483 \\
\hline \multicolumn{5}{|l|}{ Laboratory data } \\
\hline $\mathrm{WBC}\left(\times 10^{9} / \mathrm{L}\right)$ & $12.7(8.7-17.9)$ & $12.6(8.8-17.3)$ & $13.0(8.1-19.1)$ & 0.66 \\
\hline HGB (g/L) & $103.3 \pm 31.7$ & $105.6 \pm 31.4$ & $98.8 \pm 32.0$ & 0.002 \\
\hline $\mathrm{ALB}(\mathrm{g} / \mathrm{L})$ & $29.5 \pm 6.7$ & $30.2 \pm 6.6$ & $28.2 \pm 6.6$ & $<0.001$ \\
\hline CREA (umol/L) & $105.0(72.0-196.0)$ & $93.5(68.8-168.0)$ & $127.0(87.0-246.5)$ & $<0.001$ \\
\hline PCT (ng/ml) & $1.6(0.5-14.4)$ & $1.2(0.4-9.7)$ & $3.5(0.7-24.0)$ & $<0.001$ \\
\hline
\end{tabular}

Data are displayed as $n(\%)$, median (25th-75th percentiles), or mean \pm SD, unless otherwise indicated. MBG, mean blood glucose; SD, standard deviation; CV, coefficient of variation; CI, cerebral infarction; CKD, chronic kidney disease; CHD, coronary heart disease; TEN, total enteral nutrition; TPN, total parenteral nutrition; EN + PN, combined enteral nutrition and parenteral nutrition; WBC, white blood cell count; HGB, hemoglobin; ALB, albumin; CREA, creatinine; PCT, procalcitonin

mortality rate of our unit was $34,2 \%$. The most common underlying comorbidities were hypertension $428(44.7 \%)$, DM 238 (24.8\%), cerebral infarction $155(16.2 \%)$, coronary heart disease 146 (15.2\%) and chronic kidney disease 134 (14.0\%). In addition, among patients who died during hospitalization, their glycemic metrics tended to be elevated, such as CV (29.36 \pm 10.07 vs $22.14 \pm 7.58 ; p<0.001)$, MBG $(10.78 \pm 2.92$ vs $9.99 \pm 2.56 ; p<0.001)$, and the incidence of hyperglycemia $(81.7 \%$ vs $76.0 \% ; p=0.04)$ or hypoglycemia $(35.7 \%$ vs $9.7 \% ; p<0.001)$ was higher (Table 1$)$.

\section{Association between dysglycemia and mortality}

We used logistic regression to investigate the effects of the 3 domains of glucose control on mortality in DM and non-DM patients and further tightly control the confounding factors, as summarized in Table 2. Information on sex, age, APACHE II score, glycemic metrics, in ICU treatment, comorbidities, nutrition therapy and laboratory data was available for 958 patients.

In the DM group, higher levels of GV (OR, 1.09; 95\% CI, 1.04-1.13) and a higher rate of hypoglycemia (OR, 
Table 2 Associations of glycemic metrics with mortality in ICU patients with and without DM

\begin{tabular}{|c|c|c|c|c|c|}
\hline & & \multicolumn{4}{|c|}{ OR $(95 \% \mathrm{CI})$ for mortality } \\
\hline & & Crude model & Model 1 & Model 2 & Model 3 \\
\hline \multirow[t]{4}{*}{$\mathrm{DM}$} & $\mathrm{CV}$ & $1.09 *(1.04-1.13)$ & $1.07 *(1.03-1.11)$ & $1.07 *(1.02-1.11)$ & $1.06 *(1.02-1.11)$ \\
\hline & Mean BG & $1.08(0.98-1.20)$ & $1.07(0.96-1.19)$ & $1.06(0.94-1.19)$ & $1.12(0.95-1.31)$ \\
\hline & Hypoglycemia $<3.9 \mathrm{mmol} / \mathrm{L}$ & $3.01 *(1.49-6.12)$ & $2.22 *(1.04-4.77)$ & $2.10(0.97-4.58)$ & $1.18(0.49-2.83)$ \\
\hline & Hyperglycemia > $10 \mathrm{mmol} / \mathrm{L}$ & $1.31(0.67-2.55)$ & $1.08(0.52-2.24)$ & $0.93(0.43-2.00)$ & $0.42(0.14-1.22)$ \\
\hline \multirow[t]{4}{*}{ Non-DM } & $\mathrm{CV}$ & $1.11 *(1.08-1.13)$ & $1.08 *(1.06-1.11)$ & $1.08 *(1.05-1.10)$ & $1.05 *(1.02-1.08)$ \\
\hline & MBG & $1.13 *(1.06-1.20)$ & $1.07(0.99-1.14)$ & $1.03(0.95-1.11)$ & $1.06(0.96-1.15)$ \\
\hline & Hypoglycemia $<3.9 \mathrm{mmol} / \mathrm{L}$ & $6.25 *(4.19-9.33)$ & $5.09 *(3.25-8.00)$ & $4.65 *(2.93-7.39)$ & $3.12 *(1.76-5.53)$ \\
\hline & Hyperglycemia $>7.8 \mathrm{mmol} / \mathrm{L}$ & $1.42(0.97-2.10)$ & $0.97(0.62-1.50)$ & $0.89(0.56-1.43)$ & $0.87(0.48-1.58)$ \\
\hline
\end{tabular}

Model 1: adjusted for age, sex and APACHE II score

Model 2: adjusted for variables in model 1 plus comorbidities, laboratory data, insulin infusion therapy (yes/no), glucocorticoid use (yes/no), duration of ventilation (hours) and duration of hemodialysis (hours)

Model 3: model 2 adjustments plus other glucose metrics (for example, to estimate the effect of CV in DM group, we adjusted for MBG, hypoglycemia, and hyperglycemia $>10 \mathrm{mmol} / \mathrm{L}$ )

${ }^{*} p \leq 0.05$

3.01; 95\% CI, 1.49-6.12) were significantly associated with a higher risk of mortality in the initial model (crude model). The effect of GV on mortality was relatively stable after adjustments for all the confounders (model 1: 1.07, 1.03-1.11; model 2: 1.07, 1.02-1.11; model 3: 1.06, 1.02-1.11). However, the association between hypoglycemia and mortality weakened and became nonsignificant after adjusting for ICU treatment and other glucose metrics (model 2: 2.10, 0.97-4.58 [ $p=0.06]$; model 3: 1.35, $0.55-3.33[p=0.51])$. In addition, although MBG and hyperglycemia were regarded as potential risk factors for mortality, the effects of MBG and hyperglycemia on mortality were nonsignificant in all models.

In the non-DM group, the effects of GV (OR, 1.11; 95\% CI, 1.08-1.13; $p<0.001)$, MBG (OR, $1.13 ; 95 \%$ CI, $1.06-1.20 ; p<0.05)$ and hypoglycemia (OR, $6.25 ; 95 \% \mathrm{CI}$, $4.19-9.33 ; p<0.001)$ on mortality were significant in the crude model. Adjusting for disease severity and other risk factors slightly decreased the ORs for hypoglycemia (model 1: OR, 5.09; 95\% CI, 3.25-8.00; model 2: OR, 4.65; 95\% CI, 2.93-7.39) but not for GV (model 1: OR, 1.08; 95\% CI, 1.06-1.11; model 2: OR, 1.08; 95\% CI, 1.05-1.10) even after adjustment for other glycemic metrics (OR, 1.05; 95\% CI, 1.02-1.08). MBG showed a positive correlation with mortality in the initial model; however, it was nonsignificant after adjustment for basic information (sex, age, and APACHE II score). In addition, the effects of GV and hypoglycemia on mortality were attenuated yet significant after adjusting for other glycemic metrics, insulin infusion therapy, glucocorticoids use, duration of ventilation, and duration of hemodialysis. Hyperglycemia had no significant effect on mortality in all models, as in the DM group.

Of note, the effect of hypoglycemia in the non-DM group (OR, 3.12; 95\% CI, 1.76-5.53) was stronger than in the DM group (OR, 1.35; 95\% CI, 0.55-3.33) after adjustment for other confounders. However, the effect of GV on mortality was notably similar in both groups. Although hyperglycemia showed nonsignificant effects on mortality in both groups in the final logistic regression model, we found that the 3 domains had a cumulative effect on mortality (Fig. 2). The mortality rate was the lowest among patients without dysglycemia and diabetes and the highest among patients with abnormality across the 3 domains of dysglycemia and without diabetes. In addition, regardless of diabetes status, the mortality rate increased proportionally with the degree of dysglycemia.

Multivariate analysis (Table 2; Fig. 2) showed that in the non-DM group, compared to those without any domains of dysglycemia (no hyperglycemia $[\mathrm{MBG} \leq 7.8 \mathrm{mmol} / \mathrm{L}$ for non-DM and $\leq 10 \mathrm{mmol} / \mathrm{L}$ for $\mathrm{DM}$ ], no hypoglycemia episodes, and $\mathrm{CV}<20 \%$ ), the cohort with 3 domains of dysglycemia had an approximately sixfold higher mortality rate. Furthermore, these associations appeared to weaken for the DM cohort, with an approximately threefold mortality increase.

\section{Factors associated with dysglycemia}

To identify the independent factors associated with dysglycemia, we devised a cumulative logistic regression model. As summarized in Table 3 , in a multivariate analysis adjusted for sex and age, APACHE II score (adjusted odds ratio $[\mathrm{AOR}]), 1.06$; 95\% $\mathrm{CI}, 1.05-1.08)$, insulin treatment 
Fig. 2 The cumulative effect of the number of dysglycemia metrics in ICU on mortality, categorized by the presence of diabetes

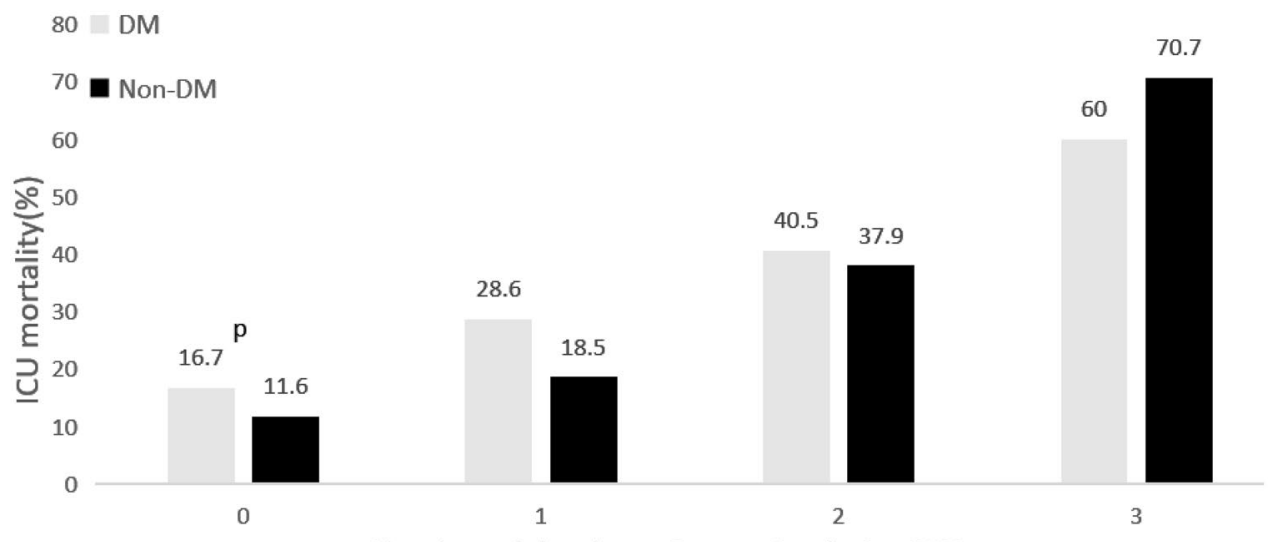

Number of dysglycemia metrics during ICU stay
(AOR, 2.50; 95\% CI, 1.91-3.28), glucocorticoid use (AOR, 1.53 ; 95\% CI, 1.18-1.98), need for mechanical ventilation (AOR, 1.94; 95\% CI, 1.43-2.64), need for renal replacement therapy (AOR, 1.99; 95\% CI, 1.48-2.67), total parenteral nutrition (AOR, 1.34; 95\% CI, 1.12-1.73), duration of diabetes (AOR, 1.04; 95\% CI, 1.01-1.08), and procalcitonin level (AOR, 1.004; 95\% CI, 1.001-1.008) were significantly associated with increasing severity of dysglycemia in critically ill patients. In contrast, patients admitted with a higher serum albumin level (AOR, 0.95; 95\% CI, 0.93-0.97) were less likely to have dysglycemia during ICU stay.

\section{Discussion}

In the present study, we investigated the association between 3 domains of glucose control and mortality in acutely ill patients depending on their diabetes status. The key findings are as follows. GV was independently associated with increased mortality among critically ill patients. Its adjusted effect on mortality was similar among patients of both diabetes groups. Hypoglycemia was independently and strongly associated with mortality in the non-DM group; however, its effect attenuated to non-significance in the DM group after adjusting for confounders. Although the effect of hyperglyce$\mathrm{mia}(\mathrm{MBG}>7.8 \mathrm{mmol} / \mathrm{L}$ for non-DM patients; $>10 \mathrm{mmol} / \mathrm{L}$ for DM patients) on ICU mortality was nonsignificant, particularly after adjustment for disease severity, the association between dysglycemia (hyperglycemia, hypoglycemia, and increased GV) and mortality was cumulative in both cohorts. Compared to patients without dysglycemia, those with the 3 types of dysglycemia had nearly 3 - and sixfold higher odds of mortality in the DM and non-DM groups, respectively. A higher APACHE II score, intravenous insulin infusion, glucocorticoid use, need for mechanical ventilation, need for renal replacement therapy, lower serum albumin level, total parenteral nutrition, longer duration of diabetes, and a higher
Table 3 Multivariate analysis of factors associated with dysglycemia in critically ill patients

\begin{tabular}{llll}
\hline $\begin{array}{l}\text { Dysglycemia as an ordinal } \\
\text { categorical variable }(0,1,\end{array}$ & OR & $95 \%$ CI & $p$ value \\
$2,3)$ & & & \\
\hline APACHE II, per 1 increment & 1.06 & $1.05-1.08$ & $<0.001$ \\
Insulin use & & & \\
No & 1 [reference] & & \\
Yes & 2.50 & $1.91-3.28$ & $<0.001$ \\
Treated with corticosteroids & & & \\
No & 1 [reference] & & \\
Yes & 1.53 & $1.18-1.98$ & $<0.001$ \\
Need for MV & & & \\
No & 1 [reference] & & \\
Yes & 1.94 & $1.43-2.64$ & $<0.001$ \\
Need for RRT & & & \\
No & 1 [reference] & & \\
Yes & 1.99 & $1.48-2.67$ & $<0.001$ \\
SA, per 1 g/L increment & 0.95 & $0.93-0.97$ & $<0.001$ \\
TPN & & & \\
No & 1 [reference] & & \\
Yes & 1.34 & $1.12-1.73$ & 0.01 \\
DOD, per 1 year increment & 1.04 & $1.01-1.08$ & 0.01 \\
PCT, per 1 ng/ml increment & 1.004 & $1.001-1.008$ & 0.02 \\
\hline
\end{tabular}

DOD, duration of diabetes (years); MBG, mean blood glucose level; MV, mechanical ventilation; RRT, renal replacement therapy; IT, insulin treatment; SA, serum albumin; TPN, total parenteral nutrition; GU, glucocorticoids use; PCT, procalcitonin

procalcitonin level were significantly associated with the 3 domains of dysglycemia in critically ill patients.

These findings support the critical role of the extended concept of glucose control, which should include the 3 dimensions (hyperglycemia, hypoglycemia, and GV) and patient diabetes status into consideration. 
Several observational studies have reported the association between dysglycemia (hyperglycemia, hypoglycemia, and GV) and mortality in patients admitted to the ICU [3, 4, 11]. In addition, recent studies have reported that associations between glycemic metrics and adverse outcomes varied among patients with or without diabetes $[7,9,11,12,14$, 21]. However, the results of these studies are inconsistent, which can partially be explained by a lack of adjustment for significant confounding factors such as comorbidities, insulin infusion, use of glucocorticoids, and hemodialysis in these studies. In our work, after adjusting for all the confounders we selected, the robust regression model showed that the effects of dysglycemia on mortality in critically ill patients differed between DM and non-DM patients.

Previous studies have suggested that GV has a more pronounced effect on mortality in patients with DM than in those without $[6,14,25,26]$. In contrast, a recent multicenter study reported that the independent effect of GV on mortality was unaffected by diabetes status or even by $\mathrm{HbA1c}$ level [27]. Consistent with this finding, our results show that GV had a similar and independent effect on mortality in both DM and non-DM groups. Its effect on mortality did not reduce significantly even after adjustment for hypoglycemia and hyperglycemia. Given studies have reported that the effect of GV on mortality can be attenuated by the interaction between hypoglycemia and mortality in the non-diabetic group [27, 28], we believe that the explanation could be our selection of relatively comprehensive confounders, including multiple glucose metrics, comorbidities, disease severity, and in ICU treatment, which were added to the regression model step by step for adjustment.

In accord with most previous studies, our study reported that hypoglycemia, defined as a minimum $\mathrm{BG}<3.9 \mathrm{mmol} / \mathrm{L}$, has the strongest effect on mortality among the 3 dimensions of glucose control and is independently associated with high mortality in critical patients without diabetes [28, 29]. In addition, results of this study showed that this deleterious effect in patients with diabetes was significant in our initial model but attenuated and turned nonsignificant after adjustment for comorbidities, ICU treatment and disease severity. Similarly, a prospective study also showed that glucose level fluctuations in patients with diabetes are more likely to be strongly associated with adverse outcomes than hypoglycemia [30]. In addition, findings from observational studies have shown that when exposed to hypoglycemia, those with higher HbA1c levels (poor glycemic control) before admission had a lower mortality rate [14].

Hyperglycemia is a well-known marker of disease severity, the association between hyperglycemia and mortality in ICU has been reported in many studies $[6,31]$. Per recent studies, the association between hyperglycemia and mortality in patients admitted to the ICU is more pronounced in patients without diabetes than in patients with diabetes $[12,32]$. Our univariate analysis of hyperglycemia $(\mathrm{MBG} \leq 7.8 \mathrm{mmol} / \mathrm{L}$ for non-DM and $\leq 10 \mathrm{mmol} / \mathrm{L}$ for $\mathrm{DM})$ was consistent with these results. However, this association attenuated to non-significance in both groups after adjustment for disease severity. A plausible explanation for this observation is that the association between hyperglycemia and mortality is most affected by acute stress response, particularly when adjusting for the effect of diabetes [33]. In line with this observation, recent studies reported that hyperglycemia is not significantly associated with mortality after adjustment for disease severity [31, 34].

Moreover, our results showed that abnormalities in $>1$ domain of glycemic control (including hyperglycemia) had a cumulative effect on mortality both in DM and non-DM groups, which is consistent with results of other studies [25].

Dysglycemia is defined as a deviation or fluctuations of blood glucose levels from the normal levels [11]. This common metabolic dysfunction in ICU could partly be explained by stress response, which involves several cellular pathways, such as those related to oxidant stress, immunity, and cellular homeostasis [35]. However, associations between diabetes status and nutritional condition and use of some medications (insulin/steroids) are complex [35] and can lead to poor glycemic control through increased insulin resistance and reduced $\beta$-cell secretory function.

Given the disadvantages of poorly controlled glycemic metrics, we believe identifying patients at high risk for manifested dysglycemia is crucial to a more personalized approach for targeted therapy. However, the factors associated with ICU dysglycemia are complex [36]. We simply divided them into endogenous factors (such as age, sex, disease severity, the function of pancreatic beta-cell) and exogenous factors (such as surgical trauma, nutrition therapy, insulin infusion therapy, and use of glucocorticoids) according to previous studies [34, 37].

After adjusting for covariates, severity of illness (marked as APACHE II score) had the strongest effect on dysglycemia among all the endogenous factors, followed by duration of diabetes. Prolonged diabetes can lead to worsening dysglycemia through increased insulin resistance and reduced $\beta$-cell secretory function [38]. Elevated procalcitonin level, used as a clinical marker for infection or inflammation in critically ill patients, was significantly associated with dysglycemia. Of interest, the negative correlation between serum albumin and dysglycemia suggested that nutritional status may probably help stabilize glycemic control.

Per our multivariate analysis, insulin use was the most critical factor determining the severity of dysglycemia, followed by renal replacement therapy and mechanical ventilation. Corticosteroid use also had a significant effect on dysglycemia. Total parenteral nutrition therapy during ICU appeared to play a relatively minor role in glycemic control 
in critically ill patients than other exogenous factors. The sample size of this study limited the evaluation of the relative effects of various dose ranges of exogenous factors on glycemic control.

Glucose level management in ICU is a key clinical concern yet the optimal glucose level target remains unclear. Our results provide further understanding of the $3 \mathrm{com}-$ mon domains of glycemic control, showing that glycemic control in critically ill patients should be in consideration of the patient's diabetes status. Considering the increasing body of evidence highlighting the varying associations between dysglycemia domains and mortality in critically ill patients, we reckon future critical illness guidelines for glycemic control targets will recommend a personalized approach [39]. Although the causal relationship between adverse outcomes and poorly controlled dysglycemia or its 3 domains remains unclear, appropriate control of glycemic metrics has been considered to beneficial for critically ill patients [17, 35, 40,41], and appropriate control of glycemic metrics is associated with better short-term and long-term outcomes. Further prospective cohort studies and randomized controlled trials are required to validate our findings.

A strength of our study is the comprehensive selection of confounding variables that allowed estimating the association between multiple glucose metrics, diabetes status, and mortality accurately, which was lacking in previous studies to our knowledge. We stratified patients into 2 groups and used 3 models adjusting for confounders, which allowed reliably estimating the effect of each glucose metric on mortality. Another strength lied in defining dysglycemia as an ordinal categorical variable, determining its cumulative effect on mortality, and further identifying its risk factors.

Our study had some limitations. First, this was a singlecenter study. Although the patient population was heterogeneous (patients admitted with a variety of medical and surgical diagnoses), it is not completely representative of all critically ill patient population.

Second, given the retrospective study design, selection bias and misclassification cannot be excluded. Because physicians in our ICUs do not routinely request $\mathrm{HbA} 1 \mathrm{c}$ measurements without a clinical suspicion of diabetes, we only used electronic medical records to identify DM status. Therefore, we could not determine whether patients with undiagnosed diabetes were included and could not further analyze stress-induced hyperglycemia and diabetes-induced hyperglycemia, which required $\mathrm{HbAlc}$ measurements [32, 33, 42].

Furthermore, we could not determine the type of DM, which is critical given that significant differences may exist between DM types. Considering a recent study focusing on higher GV in critically ill patients with type 1 DM [43], we hope further research will be conducted on personalized ICU glucose control strategy for the different diabetes types. In addition, observational studies have found that different nutritional provisions and therapies are associated with an increased risk of dysglycemia and adverse outcomes [44]. However, because of a limited scope of the medical records used in our study, the nutrition and insulin infusion data extracted were dichotomous variables used only for adjustments and further identification of risk factors of dysglycemia, we could not further determine the precise insulin dose and energy intake, which have been shown to affect dysglycemia [44]. Further research with individual-level data on these confounders may help clarify these questions.

\section{Conclusion}

This retrospective single-center observational study demonstrated that dysglycemia occurring during ICU admission was associated with mortality in all patients. However, the associations between the 3 domains of glycemic control and mortality in the ICU varied by diabetes status. Hypoglycemia had the strongest association with mortality, particularly among those without diabetes; however, $\mathrm{GV}$ was associated with mortality in ICU for all patients. Finally, the effect of hyperglycemia was not significant after adjustments for confounders. Of importance, the 3 glycemic metrics tended to have a cumulative association with mortality. Although the causal relationship between dysglycemia and mortality remained unclear, our findings have implications for current glucose control. Glucose management in critically ill patients should be specific to the patient's need considering the diabetes status and broader dimensions.

Acknowledgements The authors thank Dr. Zhigang Wang, Dr. Haiyan Yin, Director Jiewei Huang and Dr. Xiaohua Lu and Dr. Lihong Wang (all from Department of Critical Care and Department of Endocrinology and Metabolism, the First Affiliated Hospital of Jinan University) for their excellent assistance in data collection and writing guide. The authors also thank all of the involved clinicians, nurses, and technicians for dedicating their time and skill to this study.

Author contributions HMM and GY collected and analyzed data and wrote the paper. HMM and WTL conducted the study and collected data. HMM and GY conceived the study and analyzed data. ZWW and PRZ interpreted the data and revised the manuscript. PRZ and WTL are the guarantors of this work and, as such, had full access to all the data in the study and take responsibility for the integrity of the data and the accuracy of the data analysis. All authors read and approved the final manuscript.

Funding This study was funded by the Science and Technology Planning Project of Guangdong Province "Diabetes Intelligent Wearable 
Monitoring Device and Complication Prevention and Control Cloud Platform". (Grant No. 2016B010108008).

Availability of data and material The datasets used and analysed during the current study are available from the corresponding author on reasonable request.

\section{Declarations}

Conflict of interest There are no conflict of interest to declare.

Ethical approval and consent to participate The ethics review panel waived informed consent for all patients enrolled in the study. However, all study data were maintained anonymously. All authors have contributed to the creation of this manuscript for important intellectual content and read and approved the final manuscript.

Consent for publication This manuscript has not been published or presented elsewhere in part or in entirety, and is not under consideration by another journal.

Open Access This article is licensed under a Creative Commons Attribution 4.0 International License, which permits use, sharing, adaptation, distribution and reproduction in any medium or format, as long as you give appropriate credit to the original author(s) and the source, provide a link to the Creative Commons licence, and indicate if changes were made. The images or other third party material in this article are included in the article's Creative Commons licence, unless indicated otherwise in a credit line to the material. If material is not included in the article's Creative Commons licence and your intended use is not permitted by statutory regulation or exceeds the permitted use, you will need to obtain permission directly from the copyright holder. To view a copy of this licence, visit http://creativecommons.org/licenses/by/4.0/.

\section{References}

1. Whitcomb BW, Pradhan EK, Pittas AG, Roghmann M-C, Perencevich EN (2005) Impact of admission hyperglycemia on hospital mortality in various intensive care unit populations. Crit Care Med 33(12):2772-2777

2. Meynaar IA, Eslami S, Abu-Hanna A, van der Voort P, de Lange DW, de Keizer N (2012) Blood glucose amplitude variability as predictor for mortality in surgical and medical intensive care unit patients: a multicenter cohort study. J Crit Care 27(2):119-124

3. Krinsley JS (2008) Glycemic variability: a strong independent predictor of mortality in critically ill patients. Crit Care Med 36(11):3008-3013

4. Krinsley JS, Grover A (2007) Severe hypoglycemia in critically ill patients: risk factors and outcomes. Crit Care Med 35(10):2262-2267

5. Gerbaud E, Darier R, Montaudon M et al (2019) Glycemic variability is a powerful independent predictive factor of midterm major adverse cardiac events in patients with diabetes with acute coronary syndrome. Diabetes Care 42(4):674-681

6. Bellaver P, Schaeffer AF, Dullius DP, Viana MV, Leitao CB, Rech TH (2019) Association of multiple glycemic parameters at intensive care unit admission with mortality and clinical outcomes in critically ill patients. Sci Rep 9(1):18498

7. Egi M, Krinsley JS, Maurer P et al (2016) Pre-morbid glycemic control modifies the interaction between acute hypoglycemia and mortality. Intensive Care Med 42(4):562-571
8. Lanspa MJ, Krinsley JS, Hersh AM et al (2019) Percentage of time in range 70 to $139 \mathrm{mg} / \mathrm{dL}$ is associated with reduced mortality among critically ill patients receiving IV insulin infusion. Chest 156(5):878-886

9. Lin S, He W, Zeng M (2020) Association of diabetes and admission blood glucose levels with short-term outcomes in patients with critical illnesses. J Inflamm Res 13:1151-1166

10. Magee F, Bailey M, Pilcher DV, Martensson J, Bellomo R (2018) Early glycemia and mortality in critically ill septic patients: interaction with insulin-treated diabetes. J Crit Care 45:170-177

11. Plummer MP, Bellomo R, Cousins CE et al (2014) Dysglycaemia in the critically ill and the interaction of chronic and acute glycaemia with mortality. Intensive Care Med 40(7):973-980

12. Egi M, Bellomo R, Stachowski E et al (2008) Blood glucose concentration and outcome of critical illness: the impact of diabetes. Crit Care Med 36(8):2249-2255

13. Krinsley J, Meyfroidt G, van den Berghe G, Egi M, Bellomo R (2012) The impact of premorbid diabetic status on the relationship between the three domains of glycemic control and mortality in critically ill patients. Curr Opin Clin Nutr Metab Care 15(2):151-160

14. Krinsley JS, Rule P, Pappy L et al (2020) The interaction of acute and chronic glycemia on the relationship of hyperglycemia, hypoglycemia, and glucose variability to mortality in the critically ill*. Crit Care Med 48(12):1744-1751

15. Balintescu A, Mårtensson J (2019) Hemoglobin A1c and permissive hyperglycemia in patients in the intensive care unit with diabetes. Crit Care Clin 35(2):289-300

16. van Steen S, Rijkenberg S, van der Voort P, DeVries J (2019) The association of intravenous insulin and glucose infusion with intensive care unit and hospital mortality: a retrospective study. Ann Intensive Care 9(1):29

17. Mongkolpun W, Provenzano B, Preiser JC (2019) Updates in glycemic management in the hospital. Curr Diab Rep 19(11):133

18. American Diabetes Association (2021) Diabetes care in the hospital: standards of medical care in diabetes-2021. Diabetes Care 44(Supplement 1):S211-S220

19. van Zanten ARH, De Waele E, Wischmeyer PE (2019) Nutrition therapy and critical illness: practical guidance for the ICU, postICU, and long-term convalescence phases. Crit Care 23(1):368

20. Knaus WA, Draper EA, Wagner DP, Zimmerman JE (1985) APACHE II: a severity of disease classification system. Crit Care Med 13(10):818-829

21. Krinsley JS, Maurer P, Holewinski S et al (2017) Glucose control, diabetes status, and mortality in critically ill patients: the continuum from intensive care unit admission to hospital discharge. Mayo Clin Proc 92(7):1019-1029

22. Krinsley J, Preiser J, Hirsch I (2017) Safety and efficacy of personalized glycemic control in critically ill patients: a 2-year before and after interventional triAL. Endocr Pract : Off J Am Coll Endocrinol Am Assoc Clin Endocrinol 23(3):318-330

23. Lanspa M, Hirshberg E, Phillips G, Holmen J, Stoddard G, Orme J (2013) Moderate glucose control is associated with increased mortality compared with tight glucose control in critically ill patients without diabetes. Chest 143(5):1226-1234

24. Kovatchev B (2019) Glycemic variability: risk factors, assessment, and control. J Diabetes Sci Technol 13(4):627-635

25. Krinsley JS, Egi M, Kiss A et al (2013) Diabetic status and the relation of the three domains of glycemic control to mortality in critically ill patients: an international multicenter cohort study. Crit Care 17(2):R37

26. Plummer MP, Finnis ME, Horsfall M et al (2016) Prior exposure to hyperglycaemia attenuates the relationship between glycaemic variability during critical illness and mortality. Crit Care Resusc 18(3):189-197 
27. Hanna M, Balintescu A, Glassford N et al (2021) Glycemic lability index and mortality in critically ill patients-A multicenter cohort study. Acta Anaesthesiol Scand 65:1267-1275

28. Kim Y, Rajan KB, Sims SA, Wroblewski KE, Reutrakul S (2014) Impact of glycemic variability and hypoglycemia on adverse hospital outcomes in non-critically ill patients. Diabetes Res Clin Pract 103(3):437-443

29. Finfer S, Liu B, Chittock DR et al (2012) Hypoglycemia and risk of death in critically ill patients. N Engl J Med 367(12):1108-1118

30. Di Muzio F, Presello B, Glassford N et al (2016) Liberal versus conventional glucose targets in critically ill diabetic patients: an exploratory safety cohort assessment. Crit Care Med 44(9):1683-1691

31. Lee TF, Drake SM, Roberts GW et al (2020) Relative hyperglycemia is an independent determinant of in-hospital mortality in patients with critical illness. Crit Care Med 48(2):e115-e122

32. Chang M, Huang C, Liu H, Chen Y, Hsieh C (2018) Stressinduced and diabetic hyperglycemia associated with higher mortality among intensive care unit trauma patients: cross-sectional analysis of the propensity score-matched population. Int Journal Environ Res Public Health 15(5):992

33. Davidson P, Kwiatkowski CA, Wien M (2015) Management of hyperglycemia and enteral nutrition in the hospitalized patient. Nutr Clin Pract 30(5):652-659

34. Becker C, Sabang R, Nogueira Cordeiro M, Hassan I, Goldberg M, Scurlock C (2020) Hyperglycemia in medically critically ill patients: risk factors and clinical outcomes. Am J Med 133(10):e568-e574

35. Honiden S, Inzucchi SE (2015) Metabolic management during critical illness: glycemic control in the ICU. Semin Respir Crit Care Med 36(6):859-869

36. Olveira G, Abuin J, Lopez R et al (2020) Risk factors for hypoglycemia in inpatients with total parenteral nutrition and type 2 diabetes: a post hoc analysis of the insupar study. Endocr Pract 26(6):604-611
37. Chase J, Desaive T, Bohe J et al (2018) Improving glycemic control in critically ill patients: personalized care to mimic the endocrine pancreas. Critical care (London, England) 22(1):182

38. Landgraf W, Owens D, Frier B, Zhang M, Bolli G (2020) Fasting C-peptide, a biomarker for hypoglycaemia risk in insulin-naïve people with type 2 diabetes initiating basal insulin glargine 100 U/mL. Diabetes Obes Metab 22(3):315-323

39. Murphy CV, Saliba L, MacDermott J, Soe K, Dungan KM (2020) Individualizing glycemic control in the critically ill. Crit Care Nurs Q 43(1):14-27

40. Uyttendaele V, Dickson JL, Shaw GM, Desaive T, Chase JG (2017) Untangling glycaemia and mortality in critical care. Crit Care 21(1):152

41. Sharif K, Ghadir S, Jakubowicz D et al (2019) Improved outcome of patients with diabetes mellitus with good glycemic control in the cardiac intensive care unit: a retrospective study. Cardiovasc Diabetol 18(1):4

42. Lou R, Jiang L, Zhu B (2021) Effect of glycemic gap upon mortality in critically ill patients with diabetes. J Diabetes Investig. https://doi.org/10.1111/jdi.13606

43. Sechterberger M, van Steen S, Boerboom E et al (2017) Higher glucose variability in type 1 than in type 2 diabetes patients admitted to the intensive care unit: a retrospective cohort study. J Crit Care 38:300-303

44. Doola R, Greer RM, Hurford R et al (2019) Glycaemic variability and its association with enteral and parenteral nutrition in critically ill ventilated patients. Clin Nutr 38(4):1707-1712

Publisher's Note Springer Nature remains neutral with regard to jurisdictional claims in published maps and institutional affiliations. 